\title{
The technology of electromagnetic radiation danger estimation using the hardware-software module
}

\author{
Eugene Titov ${ }^{1, *}$, and Ivan Migalyov ${ }^{1}$ \\ ${ }^{1}$ Polzunov Altai State Technical University, 656038 Barnaul, Russia
}

\begin{abstract}
The article describes the principles of functioning of the hardware-software module, whose purpose is the estimation of a danger level of the combined electromagnetic field influence on a human organism. The module consists of the hardware and the software parts. The hardware part is an array of electromagnetic parameter detectors; the software part is an electromagnetic field modelling program based on OpenEMS. The module creates so-called images of electromagnetic environment danger. The results show practical applicability of the technological module for the stated purpose.
\end{abstract}

\section{Introduction}

At present, the electromagnetic radiation created by technogenic electromagnetic radiation sources is a valuable factor of danger in both domestic and industrial applications [1-9].

The research conducted [6-8] shows that the measured electromagnetic radiation levels generated by operation of various electrical equipment may drastically exceed the maximal permissible level (MPL) of electromagnetic radiation on some frequencies. That means that simultaneous influence of multiple electromagnetic radiation sources should be taken into account when considering electromagnetic field parameters.

The research should solve the problem of estimating the level of the danger of the personnel staying in the zone of the influence of an electromagnetic radiation caused by multiple sources. The results will help to choose reasonably the measures to protect the personnel; these measures will be based on the new principles of multi-frequency control of the electromagnetic field parameters.

\section{Experimental part}

There is a special hardware-software module designed to automate the electromagnetic field measurement process and to create the images of danger in the zones of influence of multiple electromagnetic radiation sources on various frequencies. The module allows to monitor the measurement results in the real time, and to analyze the danger image of electromagnetic radiation.

* Corresponding author: 888tev888@,mail.ru 
The hardware-software module consists of the following blocks:

- a measurement devices block (one possible variant is ST-01, MTM-01, P3-50, BEmeter AT-004 and P3-41 devices to measure, correspondingly, electrostatic; magnetostatic; alternate electric and magnetic fields on industrial frequency, i.e. $50 \mathrm{~Hz}$; electric and magnetic fields on radiofrequency, i.e. $30 \mathrm{kHz}-30 \mathrm{MHz}$; energy flow density on 300 $\mathrm{MHz}-300 \mathrm{GHz}$, together with AKS-1201 spectrum analyzer);

- a block of device adapters designed to connect the measurement devices to PC;

- the specialized PC software to gather and analyze the measurement results.

Figure 1 shows the connection of measurement devices (basic configuration) to a PC.

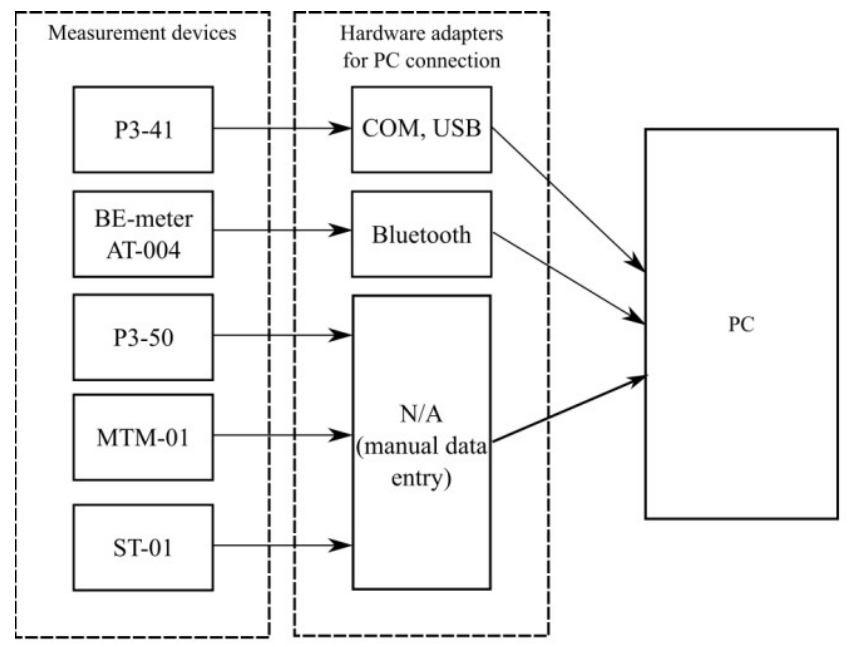

Fig. 1. Connection of measurement devices to PC.

PC saves the incoming measurement data into a separate database for every examination.

If the measurement device has the adapter that allows connecting it to the PC, it sends the experimental data directly through the adapter. Device manufacturers are not providing such adapters for some older devices, and, in that case, the hardware-software complex allows the user to enter the data manually from the PC keyboard. Existing data could also be converted to a format compatible with the complex.

After the measurement stage, the data is processed using the analytical software included into the hardware-software complex.

The functioning of the technological module is based on the following principles.

The digital model of the studied room (including all the electromagnetic radiation sources) is produced based on the geometric parameters of the facility, and the relative positions of the electromagnetic radiation sources. Every source is modeled as a $3 \mathrm{D}$ box; the model resolution should be $5 \mathrm{~cm}$.

The measurement or every controlled electromagnetic field parameter (i.e. electrical and magnetic field values, energy flow density) is performed in every of the standardized frequency diapasons (i.e. $0 \mathrm{~Hz}, 50 \mathrm{~Hz}, 30 \mathrm{kHz}-300 \mathrm{GHz}$ ), including the subdiapasons $(30 \mathrm{kHz}-3 \mathrm{MHz}, 3 \mathrm{MHz}-30 \mathrm{MHz}, 30 \mathrm{MHz}-50 \mathrm{MHz}, 50 \mathrm{MHz}-300 \mathrm{MHz}$ ) and possibly higher frequencies. The measurement should be performed on the standard distance from every face of every electromagnetic radiation source in question; the standard distance should be determined by local sanitary rules and norms for every case. The main data collected on this stage is the maximal value of every measured parameter for every accessible face of every electromagnetic radiation source in the room. 
For every frequency analyzed, prepare a computer model of the whole room, to derive the so-called electromagnetic field image for the whole room on this frequency.

AppCSXCAD program [10] used to create the 3D models allows to enter some of the electromagnetic parameters of the room on the room plan. Every electromagnetic radiation source and communication line in the room should be registered as a solid metal object.

\section{Results and discussion}

Each of the generated electromagnetic field spatial images is used to prepare the so-called electromagnetic field danger image. The hardware-software module achieves it by transforming the axe of the electromagnetic parameter (e.g. electric field, magnetic field, energy flow density) to the so-called allowed staying time (determined according to the local sanitary norms) axis in every image node.

The zones of the room where multiple danger zones are overlaying may be determined based on the analysis of the images of danger created by controlled components of electromagnetic field.

So-called cylindrical danger image is used to demonstrate the electromagnetic field danger inside of the rooms that have industrial purpose. The main difference between the point and cylindrical picture is the projection method used to prepare the picture. Every pixel of the cylindrical picture accounts the parameters of the electromagnetic field inside of the cylindrical zone (with some predetermined radius based on the industrial requirements) around the pixel. It helps to better consider the working zones of the personnel inside of the industrial room. The sample cylindrical picture of the room is presented in Figure 2.

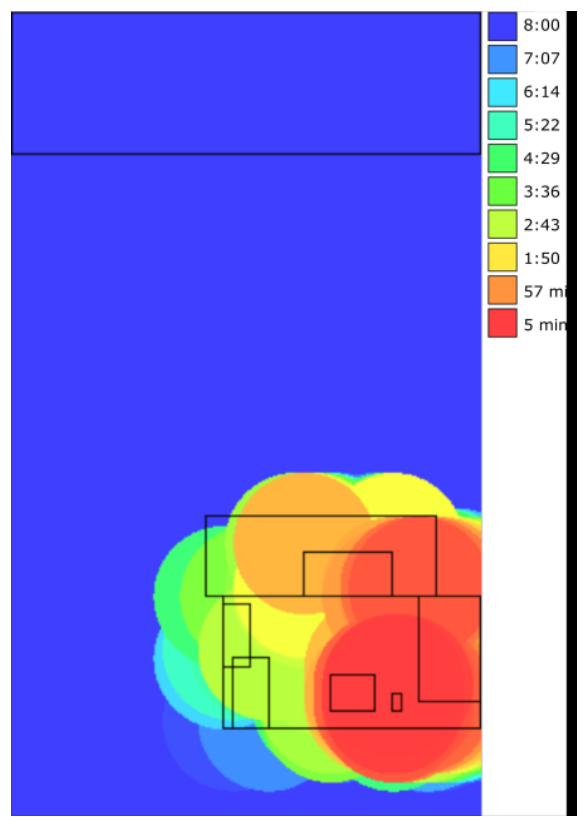

Fig. 2. Combined cylindric image of the electrolagnetic field (hours of allowed staying time). 


\section{Conclusion}

The produced technological module allows to control the danger levels in the electromagnetic environments that include multiple electromagnetic radiation sources.

For the zones of the rooms with no overlays between the danger zones, the generated images are used to prepare the protective measures based on the values of the controlled electromagnetic field components in the controlled frequency diapasons.

For the zones with intersections of multiple frequencies, a complex danger combining algorithm should be used; one perspective model for that is the danger overlay model.

The resulting danger images with refined personnel stay time with the zones of complex electromagnetic radiation influence are used to derive the protection measures for personnel with respect to frequency diapason for every frequency in the studied room.

\section{References}

1. N. A. Volodina, R. N. Karyakin, L. V. Kulikova, O. K. Nikolsky, A. A. Soshnikov, Basics of the electromagnetic compatibility (2015)

2. I. I. Davydov, Computer technology, 1 (2004)

3. T. V. Veselova, V. A. Veselovsky, L. I. Deyev, A. A. Baizhumanov, Rad.Biol. Radioec. 52(4) (2012)

4. M. A. Martínez, A. Úbeda, M. A. Cid, M. A. Trillo, Cell Physiol Biochem 29, 5 (2012)

5. World Health Organization, Electromagnetic fields and public health, URL: http://www.who.int/mediacentre/factsheets/fs322/ru/ (2007)

6. V. N. Dovbysh, M. Yu. Maslov, Yu. M. Spodobayev, Electromagnetic safety of electrical system elements: monography (2009)

7. I. E. Migalyov, Polzunovskii Vestnik, 4 (2012)

8. E. V. Titov, Increasing of agricultural complex's electrotechnology safety based on the integrated control of electromagnetic radiations: Ph. D. dissertation thesis (2013)

9. R. G. Minullin, V. I. Nazarenko, E. Yu. Zykov, and others, Hygiene and sanitation 4 (1995)

10. T. Liebig, AppCSXCAD - Minimal GUI Application using the QCSXCAD library, URL: https://github.com/thliebig/AppCSXCAD 